\title{
42. NOTES ON CRETACEOUS OSTRACODA FROM DSDP LEG 44, SITES 390 AND 392
}

\author{
F.M. Swain, Department of Geology and Geophysics, University of Minnesota, Minneapolis, Minnesota
}

\begin{abstract}
Samples from Sites 390 and 392 DSDP Leg 44, yielded, in a preliminary study, 17 species of Cretaceous Ostracoda, from preBarremian, upper Aptian, lower Albian, and upper Campanian sediments. The pre-Barremian assemblage includes one form that suggests deposition in shallow-water. The other Cretaceous ostracodes of the collection are indicative of offshore marine, but not necessarily entirely oceanic, habitats.
\end{abstract}

\section{INTRODUCTION}

I originally planned to make a more detailed study of the Mesozoic ostracodes of Leg 44 but owing to drilling difficulties I received only a limited number of samples for study of both ostracodes and carbohydrate residues of the sediments. The carbohydrates are discussed elsewhere in this volume.

In addition to the samples from which Mesozoic ostracodes were obtained the following Mesozoic samples did not yield ostracode specimens: Samples $391 \mathrm{C}-52-2,0-3 \mathrm{~cm}$ (lower Tithonian) a fissile red shale that contains poorly preserved thin-shelled gastropods? and other fossil fragments; 391C-14-3, $138-142 \mathrm{~cm}$ (upper Valanginian-early Barremian); 392A-3-1, 52-54 cm (upper Aptian); 392A-3-3, 56-68 cm (upper Aptian); 390-3-1, 116-120 cm (lower Albian); 390-4-2, 40-44 cm (lower Albian); 392A-2-3, 111-113 cm (lower Albian); $391 \mathrm{C}-7-2,132-136 \mathrm{~cm}$ (Cretaceous?).

\section{Pre-Barremian}

Sample 390-8-4, 106-110 cm (ostracodes abundant) Bairdia sp.

(Plate 2, Figures 3-5) Bythocypris? sp.

(Plate 3, Figure 2)

Aparchitocythere? sp.

(Plate 5, Figures 1-3) Xestoleberis? sp.

(Plate 7, Figures 1-4)

Rehacythereis? sp.

(Plate 6, Figures 3, 4)

Remarks: The forms resembling Aparchitocythere and Xestoleberis are the two most abundant in the collection. The former suggests deposition in shallow water, and is a genus known only from Triassic and Jurassic deposits. These types of Ostracoda occur in Upper Jurassic as well as Lower Cretaceous deposits elsewhere. Neale (written communication, 25 February 1977) has suggested relationship to Rehacythereis Grúndel, 1973. The shells of species of that genus are more pointed posteriorly than that of the present form.

\section{Upper Aptian}

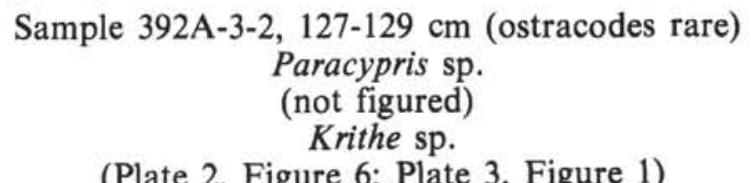

(Plate 2, Figure 6; Plate 3, Figure 1)

Remarks: The ostracodes suggest deposition in deep water.

\section{Lower Albian}

Sample 392A-2-2, 135-137 cm (ostracodes rare)

Cytherella sp. A

(not figured)

Cytheropteron $\mathrm{sp}$. aff. C. inaequivalve Bonnema

(Plate 6, Figures 5, 6)

Remarks: These forms probably lived in deep water. Neale (written communication, 25 February 1977) suggests that the Cytheropteron form resembles $C$. reightonensis Kaye, 1964, of the Barremian of England. C. reightonensis, however, is more pointed posteriorly and has more pointed aleae than the present form. $C$. inaequivalve from the Upper Cretaceous of Holland also varies in a similar way from the present species.

\section{Upper Campanian}

Sample 392A-1-2, 58-60 cm (ostracodes rare) Cytherella sp. B.

Pterygocythere sp. aff. P. laticristata (Bosquet, 1854) (Plate 4, Figures 3-5)

Phacorhabdotus? sp. aff. P.? marssoni (Bonnema, 1941)

(Plate 5, Figures 4, 5; Plate 6, Figure 2)

Remarks: These ostracodes probably lived in a deepwater habitat. Pterygocythere resembles $P$. laticristata (Bosquet) as figured by Kaye (1964) as Brachycythere laticristata from the Upper Chalk at Norwich, England. But the present species is more pointed posteriorly and has a narrow submarginal rim that is absent in P. laticristata. Phacorhabdotus? is atypical and may represent a different genus. Neale (written communication, 25 February 1977) who helpfully suggested a relationship of the present form to Cythereis marssoni Bonnema, 1941, of the upper Cretaceous Mergel of Holland thinks the species may 
be more closely related to Trachyleberidea Bowen 1953 or to Mesaleberis Deroo, 1966.

Sample 392-1-1, 117-119 cm (ostracodes common) Cytherela sp. C

(Plate 1, Figure 5)

Bairdoppilata sp. aff. B. cretacea (Veen, 1934)

(Plate 1, Figure 6; Plate 2, Figures 1, 2)

Brachycythere sp. cf. B. ledaforma Israelsky

(Plate 4, Figure 2)

Bythoceratina cf. B. bonnemai Deroo, 1966

(Plate 3, Figures 3-5)

Phacorhabdotus? sp. aff. P. ? marssoni

(Bonnema, 1941)

Remarks: The assemblage was probably deposited in deep water but whether or not it oceanic $(>7100 \mathrm{~m})$ is somewhat uncertain. Presence of some of the forms suggest that this interval is possibly Maestrichtian rather than Campanian age. I am indebted to John W. Neale for providing the comparison of the Bythoceratina with Deroo's described species, and of the Bairdoppilata with Veen's species.

\section{SUMMARY}

The Cretaceous ostracodes from the Leg 44 samples apparently represent two contrasting depth zones. The pre-Barremian assemblage contains what appears to be a shallow-water species mixed with typical offshore marine forms and perhaps were deposited on a carbonate platform bordering the ocean. The Aptian, Albian, and Campanian ostracodes of the collection probably were deposited in offshore relatively deep water, but in some instances the depths may not have been oceanic $(>7100 \mathrm{~m})$.

The Albian ostracodes are different from those described from DSDP Sites 137 and 144, Leg 14 in the central North Atlantic (Swain, 1976), but whether this is related to environment, or difference in age, cannot be determined with the material in hand.

The pre-Barremian ostracodes from Site 39 are different, for the most part, from those that Oertli (1972) found in the Jurassic at DSDP Sites 100 and 105, Leg 11. The Bairdia sp. from the pre-Barremian from Sample $390-8-4,106-110 \mathrm{~cm}$ is less elongate than either Bairdia sp. A or Bairdia sp. B of Oertli (1972, Pl. 1, fig. 1-4) and less pointed posteriorly than $B$. italica Oertli (Oertli, 1972, pl. 1, fig. 12-16). The Aparchitocythere? resembles forms known only from the Upper Jurassic of Wyoming (Swain and Peterson, 1952) that occur in shallow marine deposits.

\section{ACKNOWLEDGMENTS}

The manuscript was read by John W. Neale and Raymond H. Bate, to whom the writer is indebted for their helpful comments.

\section{REFERENCES}

Kaye, P., 1964. Revision of British marine Cretaceous Ostracoda with notes on additional forms: British Mus. Bull. (Nat. Hist.), v. 10, p. 35-79.

Oertli, H.J., 1972. Jurassic ostracoda of DSDP, Leg XI (Sites 100 and 105)-Preliminary Account. In Hollister, C.D., Ewing, J.I., et al., Initial Reports of the Deep Sea Drilling Project, Volume 11: Washington (U.S. Government Printing Office), p. 645-658.

Swain, F.M., 1976. Lower and Middle? Cretaceous Ostracoda from the Atlantic Ocean off Guiana and off West Africa: J. Paleontol., v. 50, p. 734-753.

Swain, F.M. and Peterson, J.A., 1952. Ostracodes. Ostracodes from the upper part of the Sundance Formation of South Dakota, Wyoming, and southern Montana: U.S. Geol. Survey Prof. Paper 243A, p. 1-15. 



\section{PLATE 1}

SEM Stereopairs of Cretaceous Ostracoda From Leg 44 Samples

Figure 1 Cytherella sp. B. Left valve exterior, Hole 392A, Core 1, Section 2, 58-60 cm, Upper Campanian, $\times 65$.

Figure 2 Cytherella sp. B. Left valve interior, Hole 392A, Core 1, Section 2, 58-60 cm, Upper Campanian, $\times 65$.

Figure $3 \quad$ Cytherella sp. B. Right valve, ventral view, Hole $392 \mathrm{~A}$, Core 1, Section 2, 58-60 cm, Upper Campanian, $\times 65$.

Figure $4 \quad$ Cytherella sp. C. Left valve exterior, Hole 392A, Core 1, Section 1, 117-119 cm, Upper Campanian, $\times 93$.

Figure 5 Cytherella sp. C. Left valve interior, Hole 392A, Core 1, Section 1, 117-119 cm, Upper Campanian, $\times 114$.

Figure 6 Bairdoppilata sp. aff. B. cretacea Veen, 1934, immature?. Right valve, dorsal view, Hole 392A, Core 1, Section 1, 117-119 cm, Upper Campanian, $\times 115$. 
PLATE 1
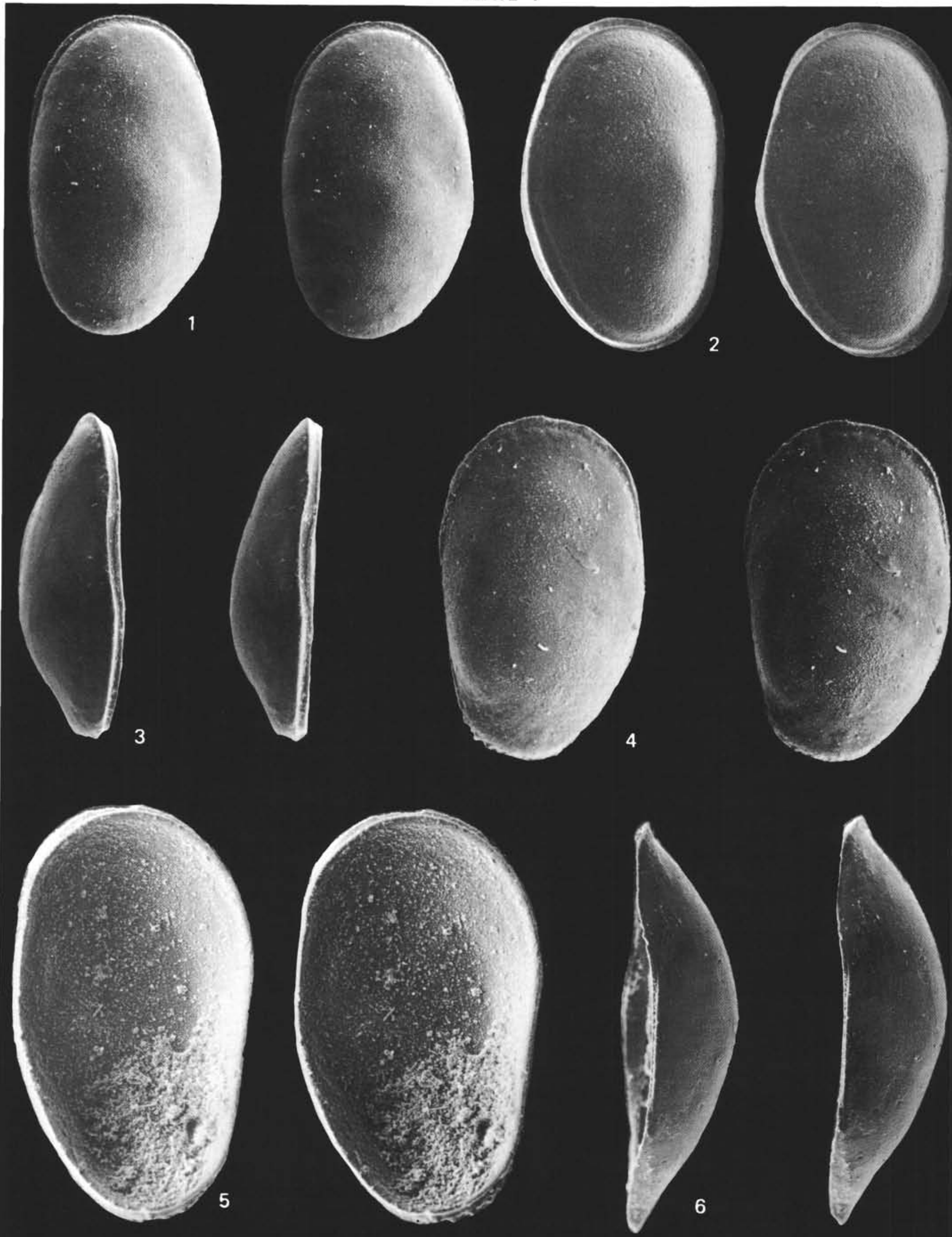


\section{PLATE 2}

SEM Stereopairs of Cretaceous Ostracoda From Leg 44 Samples

Figure $1 \quad$ Bairdoppilata sp. aff. B. cretacea (Veen, 1934). Right valve exterior, Hole 392, Core 1, Section 1, $117-119 \mathrm{~cm}$, Upper Campanian, $\times 113$.

Figure 2 Bairdoppilata sp. aff. B. cretacea (Veen, 1934). Left valve interior, Hole 392A, Core 1, Section 1, 117$119 \mathrm{~cm}$, Upper Campanian, $\times 115$.

Figure 3 Bairdia sp. Left valve exterior, Hole 390, Core 3, Section 4, 106-110 cm, pre-Barremian, $\times 58$.

Figure 4 Bairdia sp. Left valve interior, Hole 390, Core 8, Section 4, 106-110 cm, pre-Barremian, $\times 58$.

Figure 5 Bairdia sp. Dorsal view of shell, Hole 390, Core B, Section 4, 106-110 cm, pre-Barremian, $\times 58$.

Figure $6 \quad$ Krithe sp. Right valve exterior, slightly tilted toward dorsum, Hole 392A, Core 3, Section 2, 127-129 cm, Upper Aptian, $\times 104$. 
PLATE 2

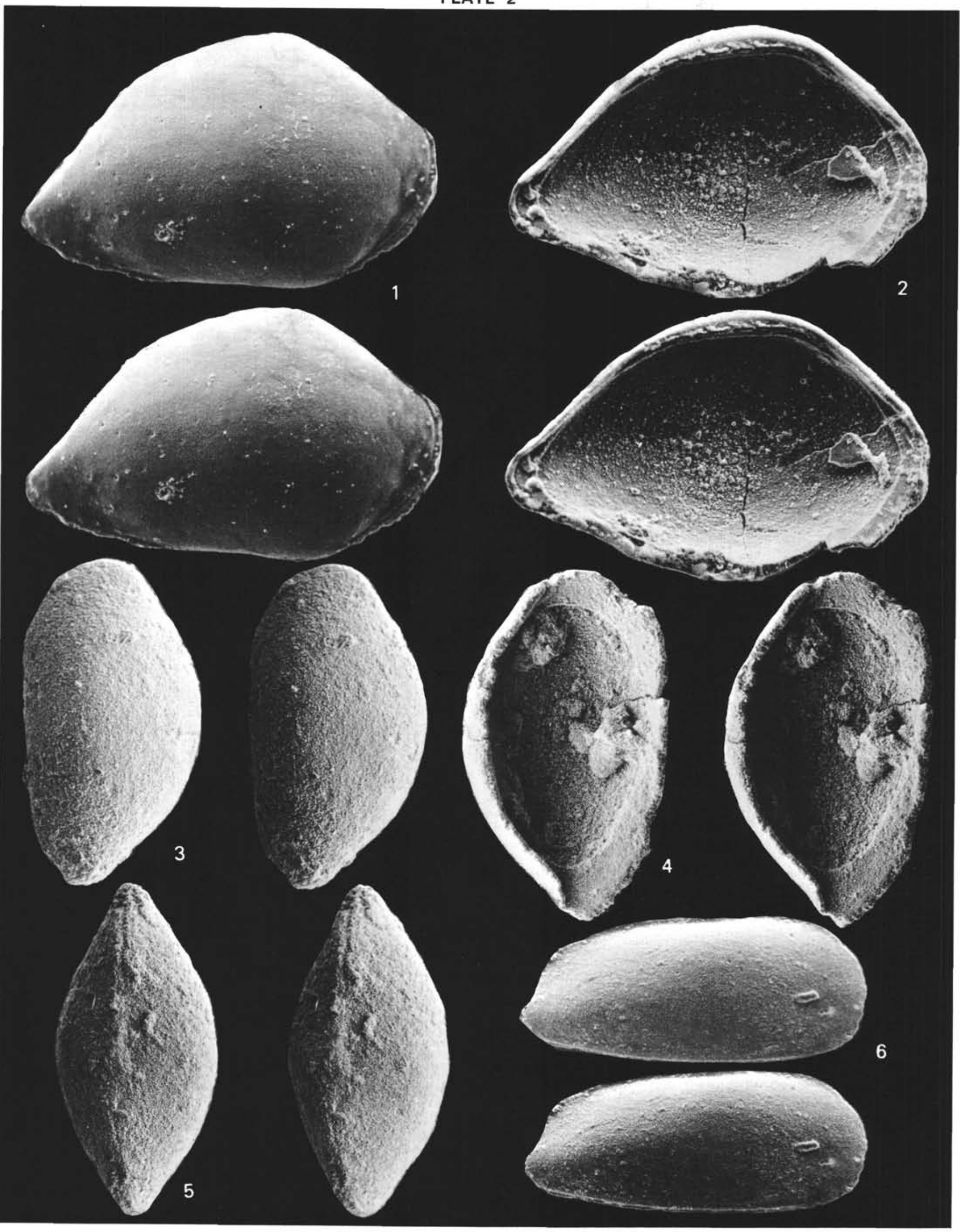




\section{PLATE 3}

SEM Stereopairs of Cretaceous Ostracods From Leg 44 Samples

Figure $1 \quad$ Krithe sp. Right valve interior, nearly filled with matrix, Hole 392A, Core 3, Section 2, $127-129 \mathrm{~cm}$, Upper Aptian, $\times 104$.

Figure 2 Bythocypris? sp. Right side of shell, having poorly preserved surface, Hole 390, Core 8, Section 4, $106-110 \mathrm{~cm}$, pre-Barremian, $\times 95$.

Figure 3 Bythoceratina $\mathrm{cf}$ B. Bonnemai Deroo. Left valve exterior, Hole 392A, Core 1, Section 1, 117-119 $\mathrm{cm}$, Upper Campanian, $\times 70$.

Figure $4 \quad$ Bythoceratina $\mathrm{cf}$ B. Bonnemai Deroo. Left valve interior, filled with matrix, Hole 392A, Core 1, Section 1, 117-119 cm, Upper Campanian, $\times 64$.

Figure $5 \quad$ Bythoceratina $\mathrm{cf}$ B. Bonnemai Deroo. Right valve, ventral view, Hole $392 \mathrm{~A}$, Core 1, Section 1, 117$119 \mathrm{~cm}$, Upper Campanian, $\times 64$.

Figure 6 Brachycythere sp. Left valve exterior, Hole 392A, Core 1, Section 1, 117-119 cm, Upper Campanian, $\times 68$. 


\section{PLATE 3}
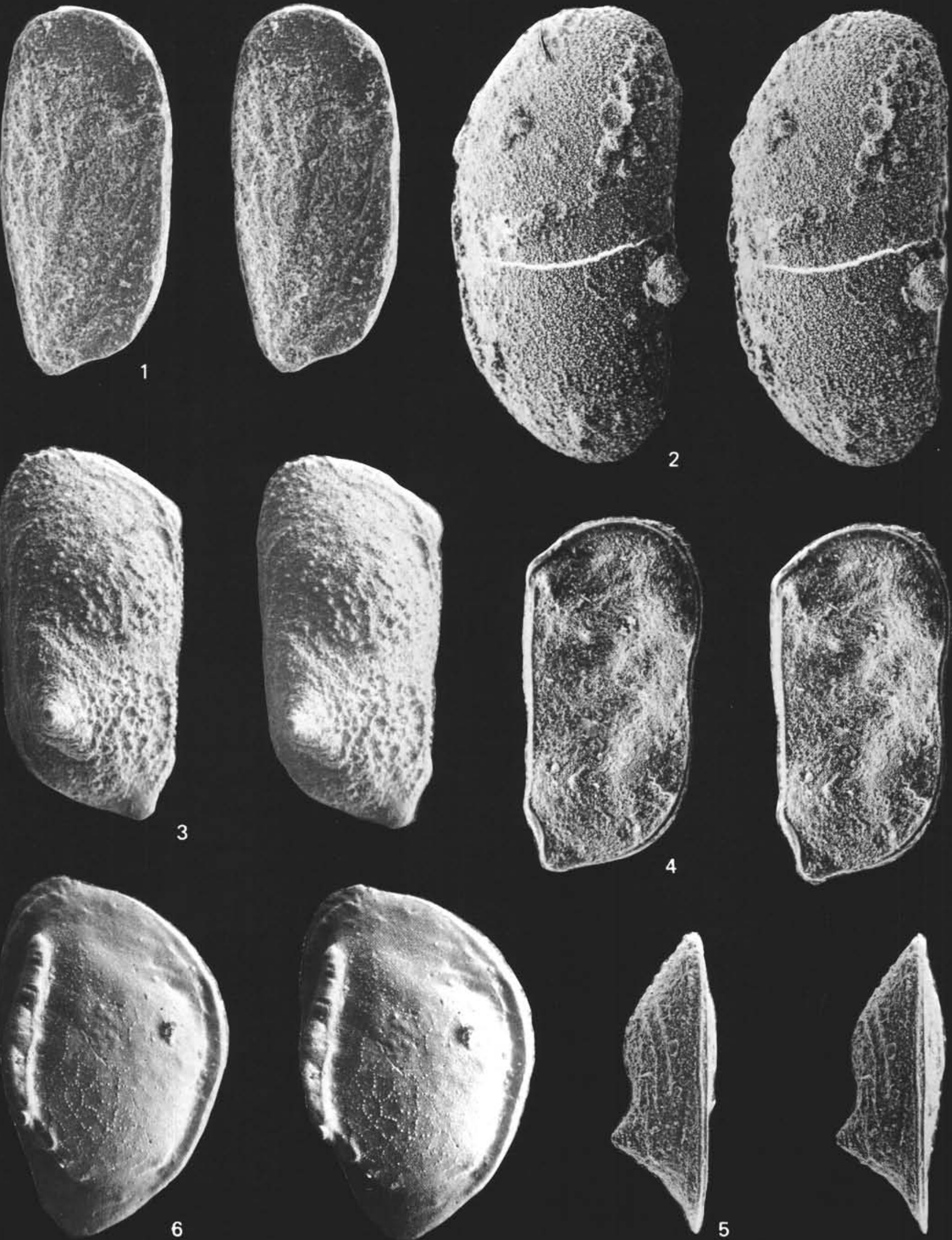


\section{PLATE 4}

Figure $1 \quad$ Brachycythere sp. Left valve, ventral view, slightly tilted, Hole 392A, Core 1, Section 1, 117-119 cm, Upper Campanian, $\times 64$.

Figure 2 Brachycythere sp. cf. B. ledaforma (Israelsky). Right valve interior, Hole 392A, Core 1, Section 1, $117-119 \mathrm{~cm}$, Upper Campanian, $\times 68$.

Figure 3 Pterygocythere sp. aff. P. laticristata (Bosquet). Left valve interior, Hole $392 \mathrm{~A}$, Core 1 , Section 2 , $58-60 \mathrm{~cm}$, Upper Campanian, $\times 64$.

Figure $4 \quad$ Pterygocythere sp. aff. P. laticristata (Bosquet). Left valve ventral view, slightly tilted, Hole 392A, Core 1, Section 2, 58-60 cm, Upper Campanian, $\times 64$.

Figure $5 \quad$ Pterygocythere sp. aff. P. laticristata (Bosquet). Left valve exterior, Hole 392A, Core 1, Section 2, 58-60 cm, Upper Campanian, $\times 64$.

Figure $6 \quad$ Brachycythere sp. Right valve, dorsal view, Hole $392 \mathrm{~A}$, Core 1, Section 1, 117-119 cm, Upper Campanian, $\times 64$. 
PLATE 4

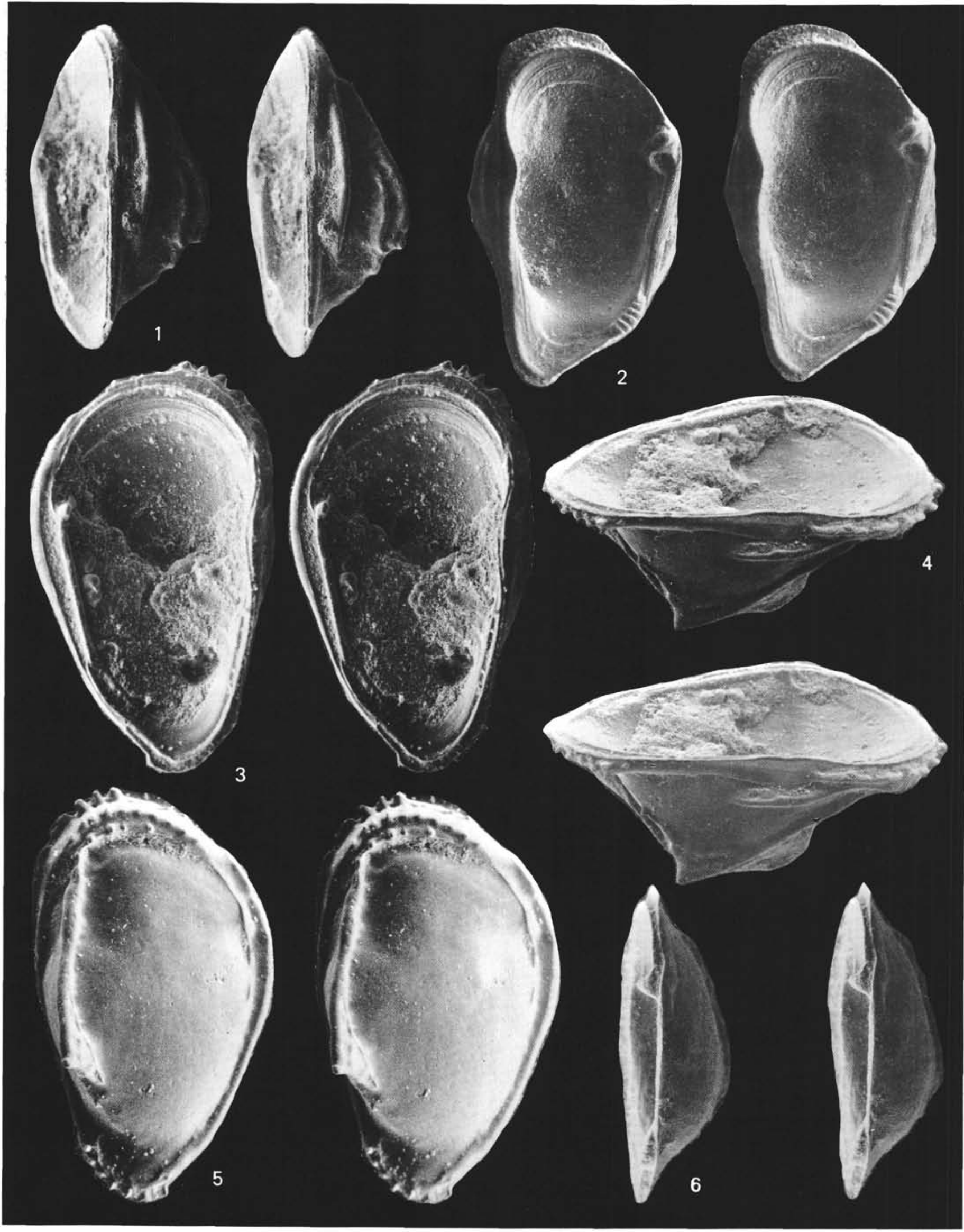




\section{PLATE 5}

Figure 1 Aparchitocythere? sp. Left side of poorly preserved shell, Hole 390, Core 8, Section 4, 106-110 cm, preBarremian, $\times 54$.

Figure 2 Aparchitocythere? sp. Dorsal view of shell, Hole 390 , Core 8, Section 4, 106-110 cm, preBarremian.

Figure 3 Aparchitocythere? sp. Right valve interior, Hole 390 , Core 8, Section 4, 106-110 cm, preBarremian, $\times 54$.

Figure $4 \quad$ Phacorhabdotus? sp. aff. P. marssoni (Bonnema). Left valve ventral view, Hole $392 \mathrm{~A}$, Core 1, Section 2, 58-60 cm, Upper Campanian, $\times 58$.

Figure $5 \quad$ Phacorhabdotus? sp. aff. P. marssoni (Bonnema). Single photograph of immature? right valve Section 2, 58-60 cm, Upper Campanian, $\times 58$.

Figure $6 \quad$ Phacorhabdotus? sp. aff. P. marssoni (Bonnema). Immature? left valve exterior, Hole 392A, Core 1, Section 1, 117-119 cm, Upper Campanian, $\times 100$. 
PLATE 5
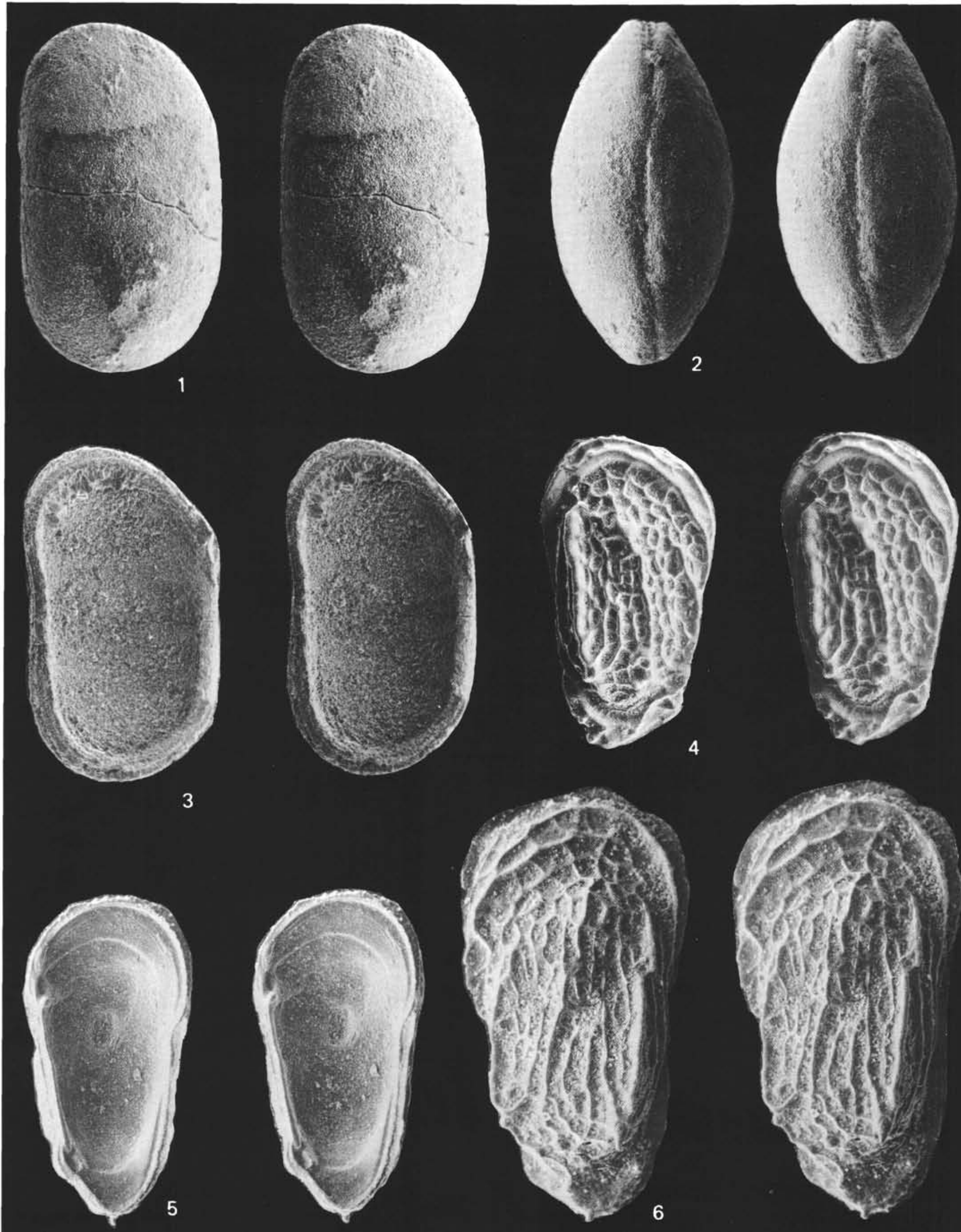


\section{PLATE 6}

Figure $1 \quad$ Phacorhabdotus? sp. aff. P. marssoni (Bonnema, 1941). Left valve ventral view, Hole 392A, Core 1, Section 2, 58-60 cm, Upper Campanian, $\times 58$.

Figure 2 Phacorhabdotus? sp. aff. P. marssoni (Bonnema, 1941). Single photograph of immature? right valve interior, Hole 392A, Core 1, Section 1, 117-119 $\mathrm{cm}$, Upper Campanian, $\times 90$.

Figure 3 Rehacythereis? sp. Left side of shell, Hole 390, Core 8, Section 4, 106-110 cm, pre-Barremian, $\times 99$.

Figure 4 Rehacythereis? sp. Ventral view of shell, Hole 390, Core 8 , Section $4,106-110 \mathrm{~cm}$, pre-Barremian, $\times 99$.

Figure $5 \quad$ Cytheropteron sp. aff. C. inaequivalve Bonnema. Left valve exterior, Hole 392A, Core 2, Section 2, 135-137 cm, Upper Albian, $\times 190$.

Figure $6 \quad$ Cytheropteron sp. aff. C. inaequivalve Bonnema. Interior of incomplete left valve, Hole 392A, Core 2, Section 135-137 cm, Lower Albian. 
PLATE 6

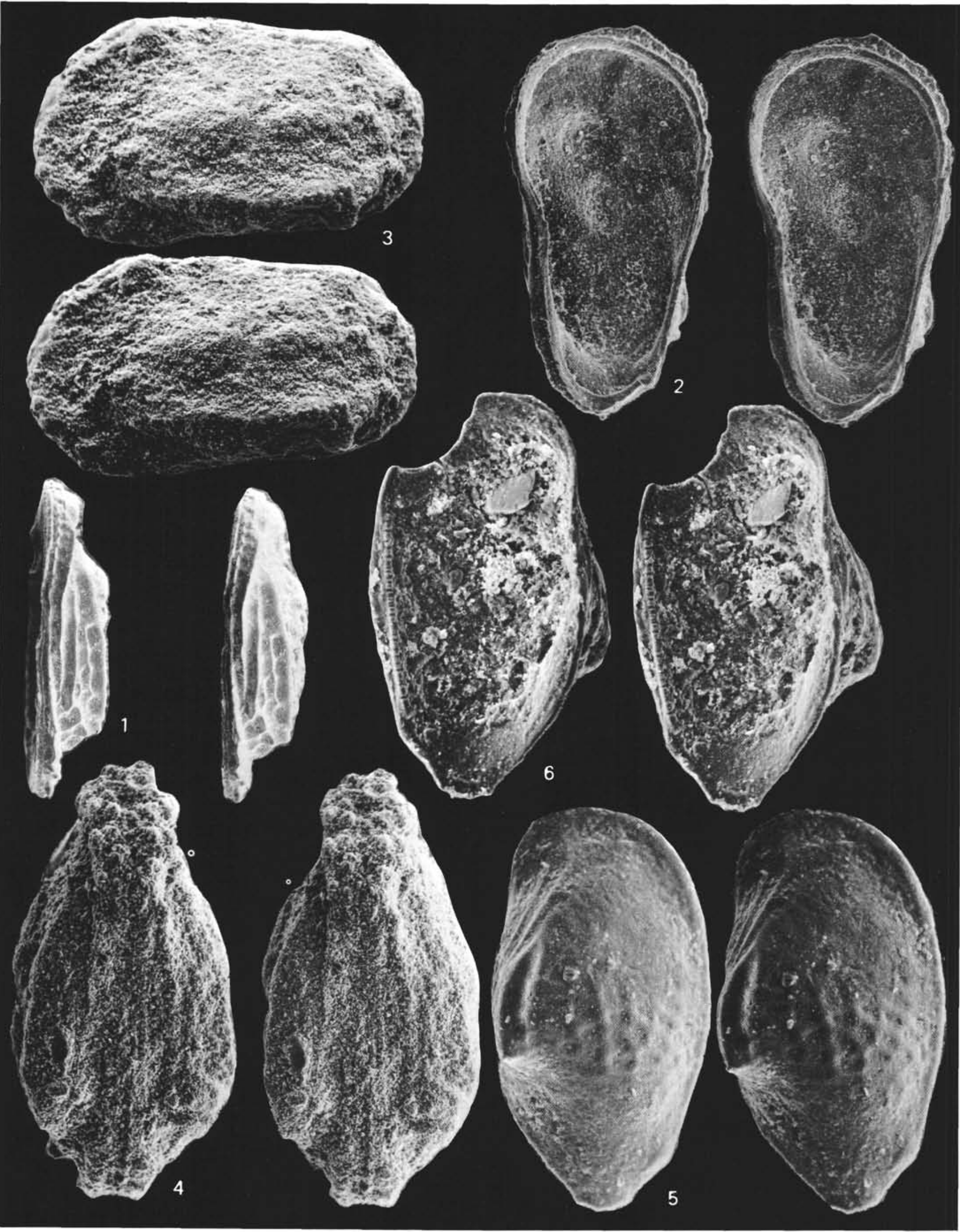


PLATE 7

SEM Stereopairs of Cretaceous Ostracoda From Leg 44 Samples

Figure 1 Xestoleberis? sp. Right side of shell, Hole 390, Core 8 , Section 4, 106-110 cm, pre-Barremian, $\times 100$.

Figure 2 Xestoleberis? sp. Ventral view of shell, Hole 390, Core 8, Section 4, 106-110 cm, pre-Barremian, $\times 100$.

Figure 3 Xestoleberis? sp. Right valve interior, Hole 390, Core 8, Section 4, 106-110 cm, pre-Barremian, $\times 100$.

Figure $4 \quad$ Xestoleberis? sp. Dorsal view of shell, Hole 390, Core 8 , Section 4, 106-110 cm, pre-Barremian, $\times 100$, 


\section{PLATE 7}

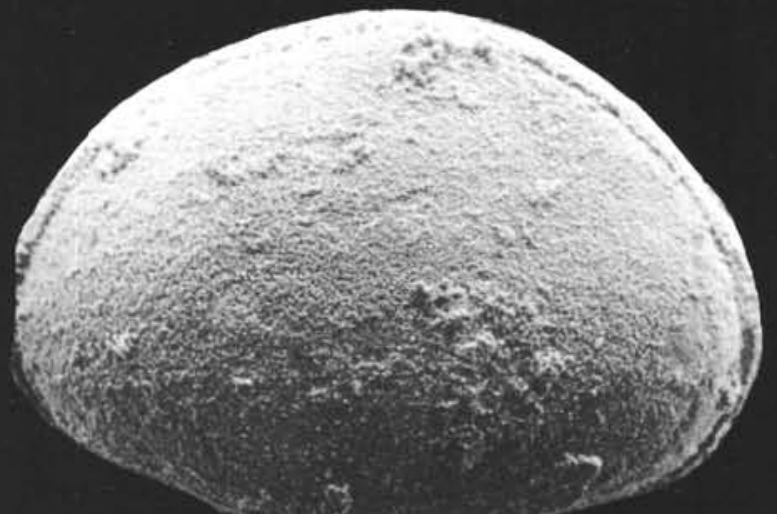

1
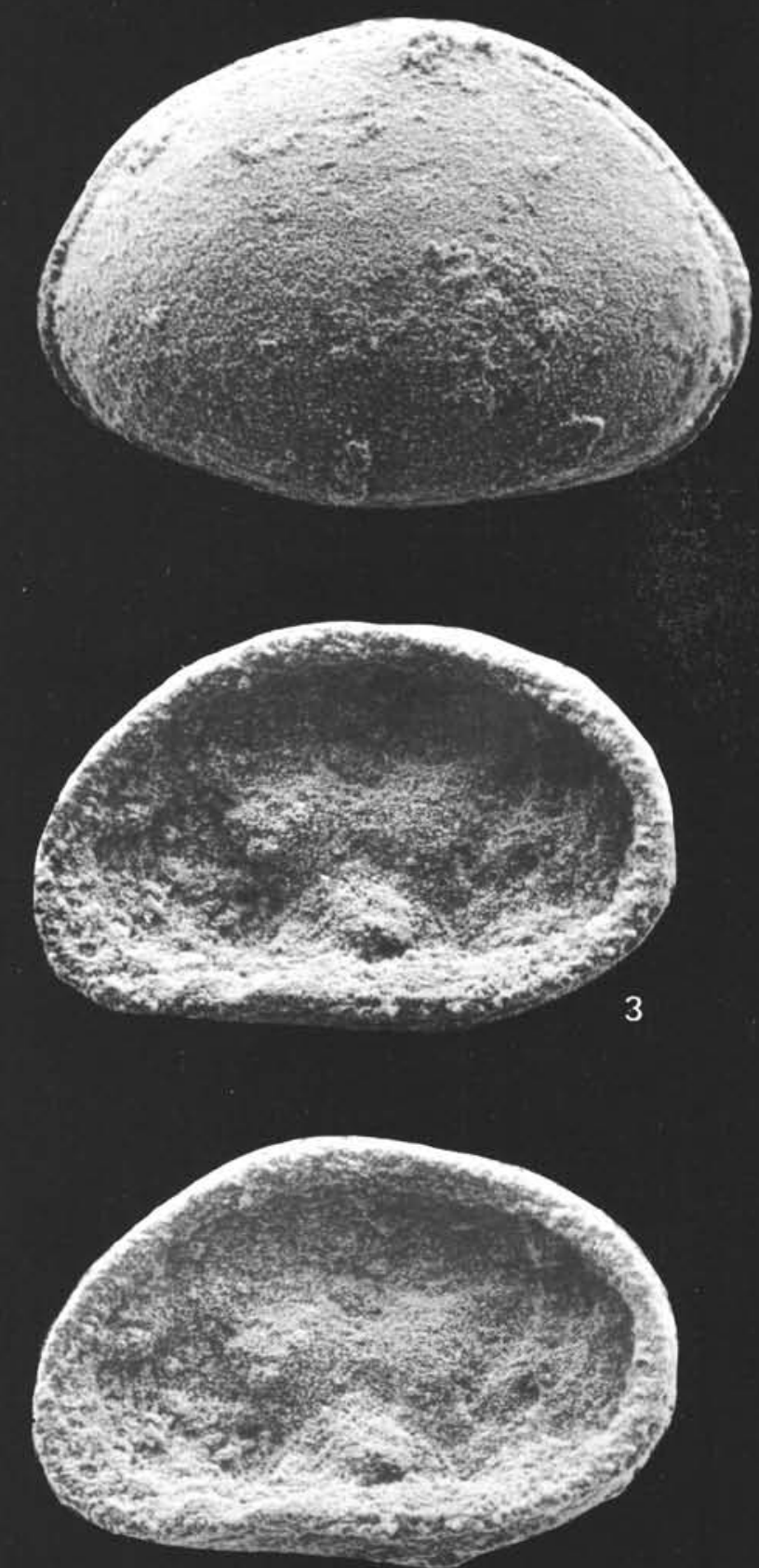
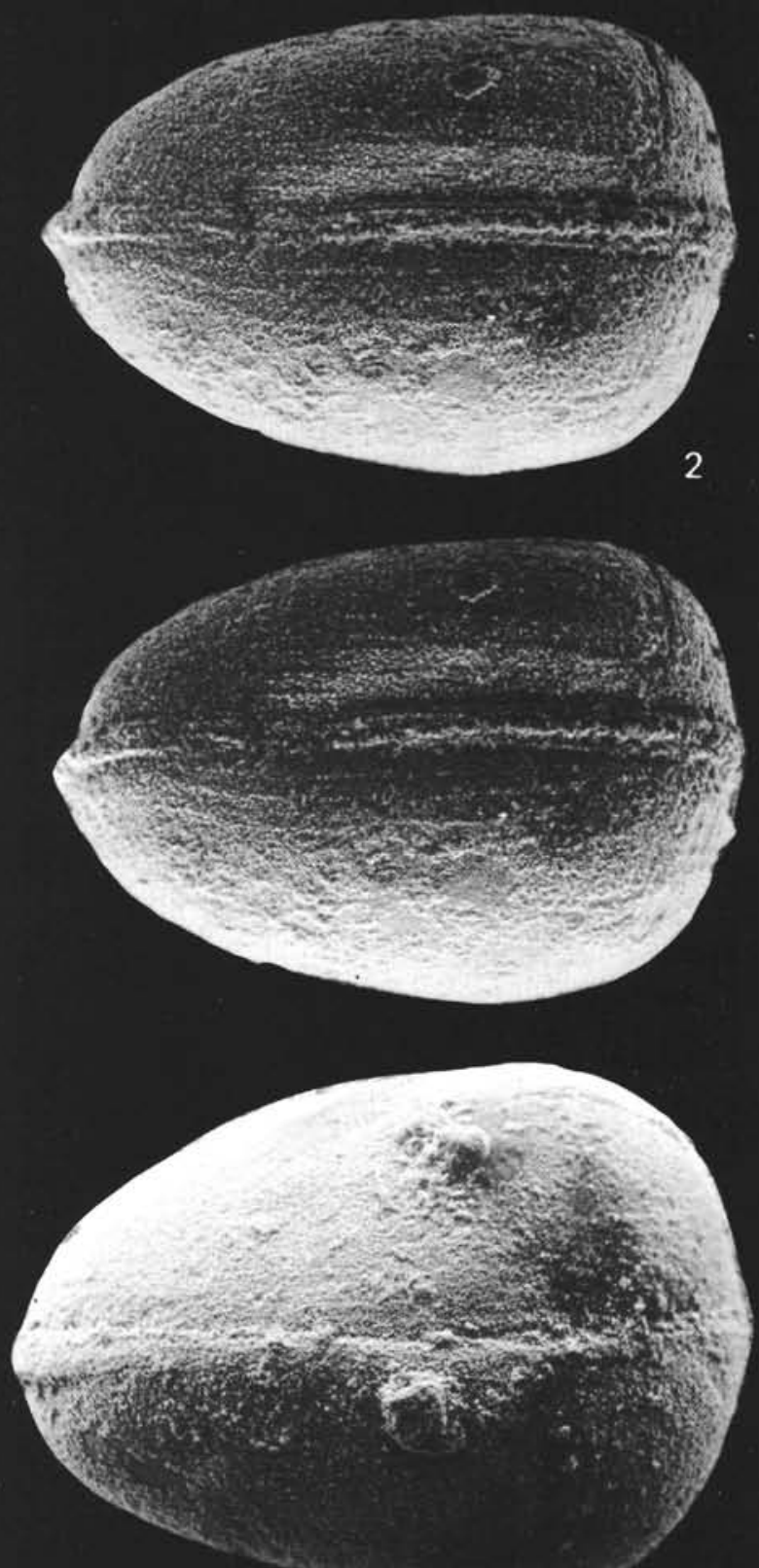

4

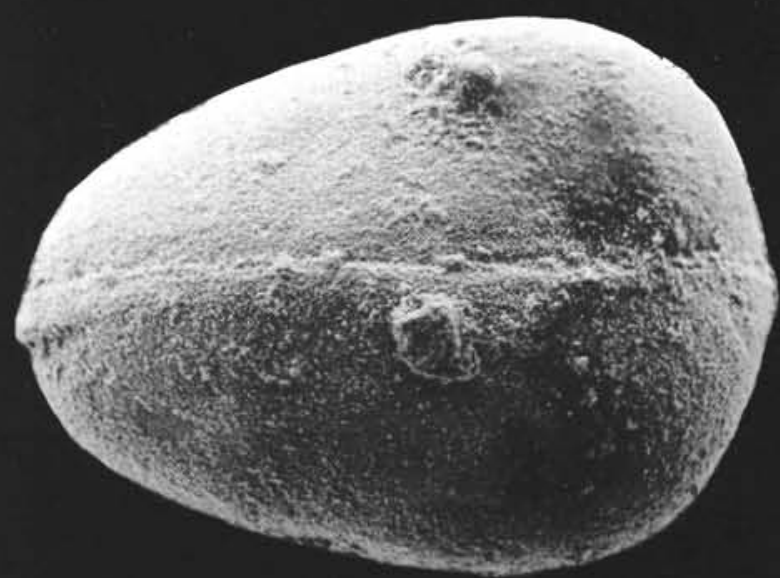

